\title{
Comparación de la carga interna y externa en competición oficial de 3 vs. 3 y 5 vs. 5 en baloncesto femenino \\ Comparison of internal and external load in official 3 vs. 3 and 5 vs. 5 female basketball competitions \\ María Reina, Javier García Rubio, Antonio Antúnez, Sergio José Ibáñez \\ Universidad de Extremadura (España)
}

Resumen. La modalidad de juego de 3 vs. 3 en baloncesto es una nueva adaptación del baloncesto convencional (5 vs. 5) en auge. El objetivo de este trabajo consistió en describir los requerimientos de carga interna y externa que demandan ambas competiciones y, en segundo lugar, analizar las posibles diferencias existentes entre ellas. Dos grupos fueron analizados. Por un lado, 10 jugadoras pertenecientes a un equipo U21, el cuál disputó ocho partidos de liga regional en la modalidad cinco contra cinco ( $\mathrm{n}=80$ ). Por otro lado, ocho jugadoras pertenecientes a dos equipos U18 que disputaron tres partidos cada uno en el campeonato de España de la modalidad de tres contra tres (n=24). Se analizaron variables de carga interna: Frecuencia cardíaca media (HRAvg), máxima (HRMáx), \% (\%HRMáx) y zonas de intensidad (Z1, Z2, Z3, Z4, Z5, Z6); y variables de carga externa: Número de pasos, saltos e impactos por minuto a través del uso de inertial devices (IMUs). Las variables de HRAvg, \%HRMáx, Z6, número de impactos y saltos por minuto fueron significativamente mayores en la modalidad de 3 vs. 3 (181,50 HRAvg frente a 171,16 ppm; 90,75 \%HRMáx frente a 84,59 \%; 85,16\% en Z6 frente a 8,10\%; 7,45 impactos por minuto frente a 1,65; y 4,50 saltos por minuto frente a 1,80). En el 5 vs. 5 se encontraron valores significativamente superiores en las zonas bajas e intermedias (Z1, Z2, Z3, Z4 y Z5) y en el número de pasos realizados por minuto (53,32 pasos por minuto frente a 44,40). Ambas modalidades tienen unas demandas diferentes en el juego y, por lo tanto, se deben prescribir entrenamientos específicos para cada modalidad, puesto que la competición también es diferente.

Palabras clave: baloncesto, modalidad de juego, carga interna, carga externa.

\begin{abstract}
Three vs. three basketball is a new adaptation of conventional basketball (five vs. five) that is currently on the rise. The purpose of this study is to describe the internal and external load requirements demanded by both competitions and, in addition, to analyze the possible differences between them. Two study groups were analyzed. On the one hand, ten players belonging to a U21 team, who played eight regional league matches in the five vs. five modality $(n=80)$. On the other hand, eight players belonging to two U18 teams who played three matches each one in the three vs. three Spanish championship competition $(n=24)$. Internal load variables: Heat Rate mean (HRAvg), maximum (HRMáx), \% (\%HRMáx) and intensity zones; and external load variables: number of steps, jumps and impacts per minute were analyzed through the use of inertial devices (IMUs). The variables of HRAvg, \% HRMáx, Z6, number of impacts and jumps were significantly higher in the 3 vs. 3 competition (181,50 HRAvg vs. 171,16 bpm; 90,75 \%HRMáx vs. 84,59 \%; $85,16 \%$ in Z6 vs. 8,10\%; 7,45 impacts per minute vs. 1,65; y 4,50 jumps per minute vs. 1,80). In 5 vs. 5 significantly higher values were found in the low and intermediate zones (Z1, Z2, Z3, Z4 and Z5) and in the number of steps performed per minute (53,32 steps per minute vs. 44,40). Both modalities had different demands in the game and, therefore, specific training must be prescribed for each modality, since the competition is also different.
\end{abstract}

Key words: basketball, play modality, internal load, external load.

\section{Introducción}

Para determinar óptimos procesos de entrenamiento se deben conocer las exigencias que provoca la competición deportiva de carácter oficial (Torres-Ronda, Ric, Llabres-Torres, de Las Heras, \& Alcazar, 2016). Por tanto, el estudio de la carga soportada por los jugadores durante partidos de competición es de vital importancia para el desarrollo y perfeccionamiento de programas de acondicionamiento físico, con el fin de optimizar el rendimiento (Sampaio, Gonçalves, Rentero, Abrantes, \& Leite, 2014). El baloncesto convencional (5 vs. 5) se define como un deporte de equipo intermitente y de alta intensidad (Drinkwater, Pyne, \& McKenna, 2008), donde se intercalan esfuerzos intensos y períodos de baja intensidad, con esfuerzos de intensidad moderada y períodos de recuperación largos (Sampaio et al., 2014).

Son muchos los estudios que consideran que tiene un carácter híbrido, en el que la mayor cantidad de energía movilizada proviene de la vía aeróbica (Abdelkrim, El Fazaa, \& El Ati, 2007; McInnes, Carlson, Jones, \& McKenna, 1995;

Fecha recepción: 23-07-19. Fecha de aceptación: 25-09-19 María Reina Román mreinarom@unex.es
Narazaki, Berg, Stergiou, \& Chen, 2009); sin embargo, al igual que ocurre en otros deportes colectivos, las acciones de carácter explosivo, tales como cambios de dirección, saltos o desplazamientos a la máxima intensidad, así como las acciones específicas de juego como los lanzamientos, los desmarques o los driblings, dependen de la vía anaeróbica y son determinantes en el rendimiento final de los deportistas (Chaouachi et al., 2009; McInnes et al., 1995; Narazaki et al., 2009; Ostojic, Mazic, \& Dikic, 2006).

La cuantificación de la carga se realiza teniendo en cuenta dos indicadores principales. La carga interna, que cuantifica las exigencias provocadas por el entrenamiento en el jugador, siendo individual y específica para cada uno de ellos. Y la carga externa, cuantifica la carga soportada por los jugadores, aquella que provoca una situación juego o tarea de entrenamiento para todos los jugadores por igual (Reina, Mancha-Triguero, García-Santos, García-Rubio \& Ibáñez, 2019). El uso de la frecuencia cardíaca como control de carga interna está aceptada y desarrollada con numerosos estudios de validación (Castagna, Impellizzeri, Chaouachi, Bordon, \& Manzi, 2011). Para la evaluación de la carga externa, el uso de inertial devices (IMUs) ha proporcionado una nueva vía de análisis de datos de forma objetiva y fiable (Reina, García-Rubio, Feu, \& Ibáñez, 2019). 
En el caso de deportes indoor, como es el caso del baloncesto, el seguimiento de las medidas de carga externa derivadas de los acelerómetros triaxiales se considera una herramienta viable (Barreira et al., 2017). Desde un punto de vista práctico, el uso de esta tecnología en las competiciones oficiales permite un control preciso de la carga que implica un partido a cada deportista en función de la modalidad, lo que permite tener a los entrenadores una referencia para estructurar el entrenamiento atendiendo a parámetros de fatiga muscular (Barrett et al., 2016). A pesar de que en la literatura analizada se encuentran multitud de datos acerca de las características del esfuerzo en baloncesto, tanto en términos de carga interna como de carga externa, se sabe más bien poco acerca de las demandas impuestas sobre el nuevo tipo de modalidad $3 \times 3$, lo que supone un problema a la hora de respetar algunos de los principios que han de marcar el entrenamiento deportivo, los de individualidad y especificidad (Bompa \& Haff, 2009; Boyle, Mahoney, \& Wallace, 1994). No se puede afirmar si las demandas generadas por la competición del 3 vs. 3 son similares a las de la modalidad convencional y, por consiguiente, se desconoce si entrenar bajo la misma metodología es lo correcto o se trata de un deporte completamente diferente en lo que a factores físicos y fisiológicos se refiere.

Por todo lo anterior, los objetivos de este estudio fueron: i) Caracterizar el perfil de carga interna y externa de jugadoras de baloncesto mediante el uso de dispositivos inerciales en partidos oficiales de 3 vs. 3 y 5 vs. 5; ii) comparar las demandas exigidas a las jugadoras en ambas competiciones. En consecuencia, se pretende aportar un mayor conocimiento sobre los requerimientos de carga interna y externa demandados en el juego reducido del 3 vs. 3 en competición. Los resultados de este trabajo permitirán a los entrenadores disponer de información relevante que deberían considerar cuando entrenen en esta nueva modalidad deportiva, siendo de gran aplicación práctica.

\section{Material y método}

\section{Diseño de investigación}

Esta investigación sigue una estrategia asociativa (Ato, López-García, \& Benavente, 2013), donde se utiliza una variable atributiva y se busca examinar las diferencias entre grupos.

\section{Población y muestra}

Se estudió la competición femenina de 3 vs. 3 y 5 vs. 5 . En cuanto a la modalidad de competición de 3 vs. 3, la investigación se llevó a cabo con los dos equipos femeninos de sub 18 (17.92 \pm 0.66 años; $54.6 \pm 3.4 \mathrm{~kg}$ y $167.3 \pm 7.2 \mathrm{~cm})$ durante el campeonato de España junior celebrado en el año 2016. Cada equipo contaba con cuatro jugadoras y disputó un total de tres partidos ( $\mathrm{n}=24$ casos). En lo que respecta a la modalidad de competición de 5 vs. 5, se analizó un equipo femenino regional sub 21 ( $19.7 \pm 3.65$ años; $59.5 \pm 12.27 \mathrm{~kg}$ y $168.5 \pm 3.56 \mathrm{~cm}$ ) de la comunidad durante la temporada 2015/ 16. El equipo contaba con un total de 10 jugadoras y disputó ocho partidos de competición oficial ( $\mathrm{n}=80$ casos). Todas las jugadoras y entrenadores fueron informados sobre el protocolo de investigación que fue aprobado por el comité de ética de la Universidad de Extremadura.

\section{Variables del estudio}

Variable Independiente: La variable independiente del estudio fue la Modalidad de Competición, con dos categorías: i) Modalidad 5 vs. 5: el partido es jugado entre dos conjuntos de cinco jugadores cada uno en pista, con la posibilidad de tener siete suplentes en banquillo. Cada partido tiene una duración de cuatro períodos de 10 minutos cada uno con tres periodos de descanso (al finalizar el primer, segundo y tercer cuarto). Las medidas de la cancha reglamentarias de la FIBA son 28 metros de largo por 15 metros de ancho; ii) Modalidad 3 vs. 3: es una adaptación del baloncesto convencional. El partido es jugado entre dos conjuntos de tres jugadores cada uno, más un jugador suplente en banquillo. Tiene una duración de 10 minutos o anotar 21 puntos antes de este tiempo, en ese caso acabará antes. En esta modalidad se disputan un mínimo de tres partidos por jornada. Se practica en una cancha de 15 metros de ancho y 11 metros de largo, lo que es equivalente a dividir la cancha del baloncesto convencional en dos, y por lo tanto sólo con un aro.

Variables Dependientes: Se seleccionaros dos grupos de variables dependientes: i) variables de carga interna (CI); ii) variables de carga externa (CE). Las variables de Carga Interna (CI): Se obtuvo mediante la Frecuencia Cardiaca(HR), la cual se establece con el número de pulsaciones. Los valores se expresarán como Frecuencia Cardíaca Media (HRAvg), se establece con la media aritmética del número de pulsaciones por minuto en un periodo de tiempo concreto (una tarea de entrenamiento o el tiempo de juego en un partido); Frecuencia Cardíaca Máxima (HRMáx), se establece con la media aritmética del número máximo de pulsaciones por minuto (ppm); \%Frecuencia cardíaca Máxima (\%HRMáx), expresa en el porcentaje de frecuencia cardiaca máxima a la que está trabajando cada deportista; y Zonas de trabajo, se dividen según el porcentaje de frecuencia cardíaca máxima que implica cada tarea de forma individual, siendo Z1 (50-60\%), Z2 (60-70\%), Z3(70-80\%), Z4(80-90\%), Z5(90-95\%) y Z6(>95\%). Las variables de carga externa (CE) se obtuvieron a partir del Número de Impactos, que miden la fuerza $G$ a la que es sometida el cuerpo en las diferentes acciones de juego (Puente, Abián-Vicén, Areces, López, \& Del Coso, 2017); el Número de Pasos, movimiento que implica avance con una elevación estándar de menos de 400 ms de vuelo; y el Número de Saltos, movimiento que consiste en elevarse de la pista con un impulso estándar que implique más de 400 ms de vuelo, para caer en el mismo lugar o en otro.

\section{Instrumentos}

Cada deportista fue equipada con una banda de Frecuencia Cardíaca de la marca GARMIN ${ }^{\mathrm{TM}}$ y un dispositivo inercial WIMU ${ }^{\mathrm{TM}}$, colocado en un arnés anatómico específico ubicado en la parte posterior del torso superior, ajustado al cuerpo. Esto se realizó antes del comienzo del partido, existiendo un período de familiarización durante el calentamiento. Durante el partido, los datos fueron visualizados en tiempo real mediante el software SVIVO ${ }^{\mathrm{TM}}$. Una vez acabado el partido se extrajeron los datos de cada dispositivo inercial y fueron analizados mediante el software SPRO ${ }^{\mathrm{TM}}$. Tanto el 
dispositivo inercial como los softwares informáticos proceden del mismo paquete de RealTrack Systems (Almería, Spain).

\section{Procedimiento}

Una vez que comienza el partido, se analiza el tiempo que cada jugadora está en pista, incluidas todas las interrupciones en el juego, excluyendo los descansos y tiempos muertos. De este modo se obtienen datos fiables y pertenecientes exclusivamente a los minutos de juego de cada jugadora. Cuando se realiza un cambio, se activa la nueva jugadora que entra al campo y se desactiva a la jugadora que se sienta. Los datos obtenidos de las variables de carga externa seleccionadas para este estudio provienen de un dispositivo inercial (que consta de acelerómetros, podómetro, radiofrecuencias, etc..), el cual no depende de sistemas de posicionamiento global.

\section{Análisis de datos}

Para el análisis estadístico, todos los datos fueron normalizados al tiempo de práctica (repeticiones por minuto), debido a que la duración de ambas competiciones es diferente. Las variables se midieron como Intensity measurement (acciones por minuto de juego). En primer lugar, se realizó un análisis exploratorio para asegurar la calidad de los datos y tipos de variables, se detectaron los valores «outliers» y se integraron todas las variables en un solo archivo. A continuación, se llevó a cabo un análisis descriptivo seguido de un clúster de las variables analizadas con el fin de caracterizar la muestra en función de la categoría escogida. Para ello, se utilizaron las medias y desviación típica. La normalidad de la muestra se testó a través de la prueba de Shapiro-Wilks. Las diferencias entre cada tipo de competición en función de las variables de carga interna y externa se analizaron mediante la prueba $T$ para muestras independientes (Field, 2009). El tamaño del efecto fue calculado a partir de la $d$ de Cohen dónde se considera un tamaño del efecto pequeño 0.20 , mediano 0.50 y grande 0.80 (Thalheimer and Cook, 2002). Finalmente, se calculó la potencia observada. Esto permite entender adecuadamente los resultados de los análisis realizados mediante una prueba relevante para la validez del estudio (Castro \& Martini, 2016). El análisis estadístico se llevó a cabo con el programa informático SPSS 25 (IBM Corp. Released 2017. IBM SPSS Statistics for Windows, Version 25.0.Armonk, NY:IBM Corp.)

\section{Resultados}

En la tabla 1, se observan los resultados del análisis descriptivo de las variables de carga interna y externa en función del tipo de competición (3 vs. 3 y 5 vs. 5). Estos resultados permiten caracterizar como son cada una de las competiciones en baloncesto femenino, observando a su vez las variaciones entre uno y otro tipo de modalidad.

Si se analizan las variables que identifican una mayor intensidad de carga interna en el juego se puede observar cómo el \%HRMáx y el \% de tiempo en la zona de mayor intensidad (Z6) son superiores en la modalidad de 3 vs. 3 , siendo este último muy significativo. En cuanto a las variables de carga externa, en la modalidad de 5 vs. 5, al utilizarse

\begin{tabular}{|c|c|c|c|c|c|}
\hline \multicolumn{6}{|c|}{5 vs. 5} \\
\hline & Variables & Media & SD & Media & SD \\
\hline \multirow{9}{*}{ Carga Interna } & HRMáx & 193,00 & 3,96 & 193,59 & 4,25 \\
\hline & HRAvg & 181,50 & 7,56 & 171,16 & 8,27 \\
\hline & \%HRMáx & 90,75 & 3,78 & 84,59 & 6,99 \\
\hline & Z1 (50-60\%) & 0,00 & 0,00 & 3,66 & 11,55 \\
\hline & $\mathrm{Z} 2(60-70 \%)$ & 0,07 & 0,21 & 6,30 & 8,13 \\
\hline & Z3 (70-80\%) & 2,92 & 6,16 & 12,35 & 6,15 \\
\hline & Z4 (80-90\%) & 6,44 & 9,89 & 37,74 & 14,79 \\
\hline & Z5 (90-95\%) & 5,41 & 5,80 & 31,85 & 14,04 \\
\hline & Z6 (>95\%) & 85,16 & 13,76 & 8,10 & 10,14 \\
\hline \multirow{3}{*}{ Carga Externa } & Impactos/min & 7,45 & 3,40 & 1,65 & 1,66 \\
\hline & Pasos/min & 44,40 & 9,78 & 53,32 & 10,17 \\
\hline & Saltos/min & 4,50 & 1,90 & 1,80 & 0,97 \\
\hline \multicolumn{6}{|c|}{$\begin{array}{l}\text { Tabla } 2 . \\
\text { Diferencias en la CI y CE entre la competición de } 3 \text { vs. } 3 \text { y } 5 \text { vs. } 5\end{array}$} \\
\hline & & \\
\hline \multirow{9}{*}{ Carga Interna } & HRMáx & $-0,521$ & 604 & 0.141 & ,266 \\
\hline & HRAvg & 4,691 & ,000 & $* \quad 1,274$ & ,894 \\
\hline & \%HRMáx & 3,561 & 001 & $* \quad 0,961$ & 1,000 \\
\hline & Z1 (50-60\%) & $-1,339$ & 022 & $* \quad 0,360$ & 1,000 \\
\hline & Z2 (60-70\%) & $-3,238$ & ,000 & $* \quad 0,871$ & 1,000 \\
\hline & Z3 (70-80\%) & $-5,639$ & , 000 & * 1,533 & 1,000 \\
\hline & Z4 (80-90\%) & $-8,366$ & 000 & $* \quad 2,262$ & 1,000 \\
\hline & Z5 (90-95\%) & $-7,746$ & 000 & $* \quad 2,089$ & ,880 \\
\hline & Z6 (>95\%) & 25,532 & 000 & $* \quad 6,967$ & 1,000 \\
\hline \multirow{3}{*}{ Carga Externa } & Impactos/min & 9,673 & ,000 & $* \quad 2,664$ & ,266 \\
\hline & Pasos/min & $-3,260$ & ,002 & $* \quad 0,885$ & ,894 \\
\hline & Saltos/min & 7,921 & , 000 & $* \quad 2,174$ & 1,000 \\
\hline
\end{tabular}

el campo completo, se realizan un mayor número de pasos por minuto, mientras que las variables que definen un carácter más explosivo (impactos y saltos) son superiores en el 3 vs. 3.

En segundo lugar, se establece la existencia o no de diferencias significativas entre las variables analizadas y la modalidad de juego (Tabla 2).

Se han encontrado diferencias estadísticamente significativas en todas las variables analizadas a excepción de la HRMáx ( $<$.005). Las variables de HRAvg, \%HRMáx, Z6, número de impactos y saltos fueron significativamente mayores en la modalidad de 3 vs. 3 . En el 5 vs. 5 se encontraron valores significativamente superiores en las zonas bajas e intermedias (Z1, Z2, Z3, Z4 y Z5) y en el número de pasos realizados por minuto. En el caso de la potencia estadística se encuentran valores óptimos en las variables en las que existen diferencias significativas (>.80) a excepción del número de Impactos por minuto. En cuanto al tamaño del efecto, se observó un efecto grande (>.80) en todas las variables dónde se identificaron diferencias estadísticamente significativas a excepción de la Zona de menor intensidad (Z1).

\section{Discusión}

La presente investigación es la primera en analizar la carga soportada por jugadoras de baloncesto durante los juegos de 3 vs. 3 y 5 vs. 5 en partidos oficiales de competición, combinando un análisis de carga interna y externa mediante el uso de dispositivos inerciales de última generación. El objetivo de este trabajo fue caracterizar la competición deportiva del baloncesto femenino en la modalidad de 3 vs. 3 y 5 vs. 5 de forma independiente y, a su vez, analizar las diferencias existentes entre ambas modalidades de competición. Entre ellas, se encontró una mayor demanda en la CI y la CE en el baloncesto de 3 vs. 3 . Se observaron diferencias estadísticamente significativas en la mayor parte de las variables analizadas, encontrando los valores más altos en el juego de 3 vs. 3 respecto al 5 vs. 5, motivado fundamentalmente, por una mayor participación de las jugadoras en la competición con menor número de participantes. 
Desde el punto de vista del entrenamiento es útil saber si la carga de trabajo ha estado por debajo o por encima de las cargas de referencia del juego real de acuerdo con las necesidades individuales. Los jugadores durante el entrenamiento deben experimentar unas demandas similares a las de la competición, por lo que los entrenadores deben conocerlas y ser capaces de reproducirlas (Conte et al., 2015; Matthew \& Delextrat, 2009; Tee, Lambert, \& Coopoo, 2016; TorresRonda et al., 2016; White \& MacFarlane, 2015). Investigaciones previas ya han demostrado que el gasto de energía, la distancia recorrida y los valores de velocidad podrían aportar una información real sobre las exigencias físicas impuestas a los jugadores (Gaudino, Alberti \& Iaia, 2014; Hodgson, Akenhead \& Thomas, 2014).

En cuanto a las variables de CI, se ha encontrado una mayor exigencia fisiológica en la modalidad de 3 vs. 3, dónde las jugadoras pasan un 85,16\% del tiempo de juego en Z6. Montgomery, Pyne y Minahan (2010) destacaron que en los partidos de 3 vs. 3 se obtiene una frecuencia cardíaca significativamente mayor y un \% de la frecuencia cardíaca máxima más elevado. Por su parte, McCormick et al. (2012) realizaron un análisis comparando situaciones de 3 vs. 3 y 5 vs. 5 en entrenamiento, dónde establecieron que no existían diferencias en actividades de modera y vigorosa intensidad en función de la situación de juego. Sampaio, Abrantes y Leite (2009), tampoco encuentran diferencias en la frecuencia cardíaca entre las situaciones 3 vs. 3 y 4 vs. 4 en entrenamiento, puesto que promueven altas demandas fisiológicas de entorno al 80\% de la frecuencia cardíaca máxima en ambas situaciones. Por tanto, se encuentran diferencias cuando se analizan ambas situaciones en competición oficial pero no cuando se analizan durante el entrenamiento, principalmente motivada por la labor del entrenador, capaz de modificar las tareas adecuándolas a las exigencias a las que quiere exponer a sus deportistas (Hernández, Casamichana, y Sánchez-Sánchez, 2017). Sin embargo, durante la competición los jugadores se exponen de una forma ecológica a las demandas generadas por la misma y es en esa situación dónde las demandas fisiológicas difieren (Reina et al., 2019).

En cuanto a las variables de CE, los datos de los indicadores globales muestran un mayor número de impactos y saltos por minuto por parte de las jugadoras en el juego de 3 vs. 3 en comparación con el 5 vs. 5, mientras que tiene lugar un mayor número de pasos/minuto en competición 5 vs. 5. Lo mismo ocurre en otras modalidades como el fútbol dónde al comparar demandas de carrera de fútbol 7 frente a fútbol 11, se obtienen valores superiores en el último (Ferrera, Sánchez, Cabrera, Sánchez, \& Moreno-Arrones, 2014). En el caso las acciones de más intensidad, Metzler (2017) también afirma que los juegos modificados (en este estudio 3 vs. 3) contienen más repeticiones en el juego debido a la menor cantidad de jugadores que, indirectamente, ofreció a los jugadores más espacio y tiempo para tomar decisiones y ejecutarlas (López-Herrero \& Arias-Estero, 2019). Estos resultados coinciden también con los descritos por Sampaio et al. (2009), que indican que la reducción de jugadores en el mismo espacio provoca una mayor participación de los mismos, incrementando la intensidad en las acciones (Coutinho et al 2019).

En cuanto al tamaño del terreno de juego, estudios pre- vios han demostrado que este aspecto puede ser un factor influyente en la respuesta fisiológica durante el entrenamiento. Por ejemplo, Hill-Haas, Coutts, Rowsell, \& Dawson (2009) informaron que variar el área total de juego influye en la intensidad de la tarea, siendo superior en juegos reducidos (3 vs. 3) realizados en una superficie de juego mayor (campo completo). La competición de 5 vs. 5 se realiza en un campo convencional y la competición de 3 vs. 3 se realiza en la mitad de ese mismo campo. Por tanto, el mayor número de pasos obtenidos en la modalidad de 5 vs. 5 puede ser debido principalmente al desplazamiento realizado de un lado del campo a otro. Sin embargo, en el 3 vs. 3 se dio lugar un mayor número de acciones de alta intensidad y una respuesta fisiológica superior, principalmente debido al aumento de la ratio de espacio por parte de los jugadores

Pasar de jugar de 5 vs. 5 a 3 vs. 3 no es simplemente pasar de jugar de pista completa a media pista. Es necesario que los jugadores se familiaricen con el nuevo espacio de juego, el número de compañeros y la orientación en el campo. Hay que proponer entrenamientos específicos para competir en la nueva modalidad de juego, las tareas deben simular el mismo escenario perceptivo-motriz que las jugadoras se van a encontrar en la competición real (Olthof et al., 2017; Travassos et al., 2012). Para una futura adaptación al entrenamiento, es importante tener en cuenta que en el caso de la modalidad de 3 vs. 3 , pueden disputarse más de un encuentro en el mismo día, por lo que la carga soportada por parte de las jugadoras sería superior al finalizar la jornada. Además, se deben considerar las diferencias individuales entre jugadores, género, partidos y equipos. Sin embargo, estos datos permiten hacer una primera reflexión general y recopilar una serie de contribuciones y aplicaciones para la capacitación por parte de entrenadores.

\section{Fortalezas y limitaciones del estudio}

Con los resultados obtenidos por este estudio, se ha podido identificar que las demandas generadas por ambas competiciones son diferentes, pudiendo afirmar que, aun manteniendo en ambas modalidades la esencia del juego del baloncesto, se tratan de dos deportes energéticamente distintos. Por otra parte, se establece como limitación que la muestra en cada una de las modalidades es diferente. Aún así, los grupos de edad son similares y permite hacer una primera caracterización y comparación de ambas modalidades.

\section{Conclusiones}

El análisis de la CI y CE en competición oficial de baloncesto de 3 vs. 3 y 5 vs. 5 a través de dispositivos inerciales ha servido de gran ayuda para conocer las demandas de ambas modalidades de manera válida y fiable. Tras describir los requerimientos de ambas competiciones, se puede concluir que las modalidades competitivas analizadas en este trabajo presentan demandas diferentes, siendo más intensa la modalidad reducida de 3 vs. 3 . Los resultados de este estudio permiten a los entrenadores obtener información relevante a la hora de diseñar entrenamientos específicos en función de la modalidad. En el caso de la modalidad emergente de 3 vs. 
3 se extrajeron conclusiones prácticas tales como: sesiones de entrenamiento más cortas, pero más intensas, tareas de entrenamiento con descansos activos, frecuencia cardíaca constante y en zonas altas, uso de medias canchas únicamente, y el aumento del número de impactos y saltos durante los entrenamientos.

\section{Agradecimientos}

Este trabajo ha sido parcialmente subvencionado por la Ayuda a los Grupos de Investigación (GR18170) de la Junta de Extremadura (Consejería de Economía e Infraestructuras); con la aportación de la Unión Europea a través de los FEDER y con la colaboración de la Federación Extremeña de Baloncesto.

\section{Referencias}

Abdelkrim, N. B., El Fazaa, S., \& El Ati, J.(2007). Time-motion analysis and physiological data of elite under-19-yearold basketball players during competition. British journal of sports medicine, 41(2), 69-75.

Ato, M., López-García, J. J., \& Benavente, A. (2013). Un sistema de clasificación de los diseños de investigación en psicología. Anales de Psicología, 29(3), 1038-1059.

Barreira, P.; Robinson, M.A.; Drust, B., Nedergaard, N.; Raja Azidin, R. M. F., \& Vanrenterghem, J. (2017). Mechanical Player LoadTM using trunk-mounted accelerometry in football: Is it a reliable, task- and player-specific observation? Journal of Sports Sciences, 35(17), 1674 1681.https://doi.org/10.1080/02640414.2016.1229015

Barrett, S., Midgley, A. W., Towlson, C., Garrett, A., Portas, M., \& Lovell, R. (2016). Within-match PlayerLoad ${ }^{\mathrm{TM}}$ patterns during a simulated soccer match: Potential implications for unit positioning and fatigue management. International Journal of Sports Physiology and Performance, 11(1), 135-140.

Bompa, T. O., \& Haff, G. G. (2009). Periodization: Theory and methodology of training: Human Kinetics Publishers.

Boyle, P., Mahoney, C., \& Wallace, W. (1994). The competitive demands of elite male field hockey. The Journal of Sports Medicine and Physical Fitness, 34(3), 235-241.

Buchheit, M., Laursen, P., Kuhnle, J., Ruch, D., Renaud, C., \& Ahmaidi, S. (2009). Game-based training in young elite handball players. International Journal of Sports Medicine, 30(4), 251.

Castagna, C.; Impellizzeri, F. M.; Chaouachi, A.; Bordon, C., \& Manzi, V. (2011). Effect of Training Intensity Distribution on Aerobic Fitness Variables in Elite Soccer Players: A Case Study. Journal of Strength and Conditioning Research, 25(1), 66-71. https://doi.org/ 10.1519/JSC.0b013e3181fef3d3

Conte, D., Favero, T. G., Lupo, C., Francioni, F. M., Capranica, L., \& Tessitore, A. (2015). Time-Motion Analysis of Italian Elite Women's Basketball Games: Individual and Team Analyses. The Journal of Strength \& Conditioning Research, 29(1), 144-150.

Coutinho, D., Gonçalves, B., Santos, S., Travassos, B., Wong, D. P., \& Sampaio, J. (2019). Effects of the pitch configuration design on players' physical performance and movement behaviour during soccer small-sided games. Research in Sports Medicine, 27(3), 298-313.

Chaouachi, A., Brughelli, M., Levin, G., Boudhina, N. B. B., Cronin, J., \& Chamari, K. (2009). Anthropometric, physiological and performance characteristics of elite team-handball players. Journal of Sports Sciences, 27(2), 151-157.

Delextrat, A., Badiella,A., Saavedra, V., Matthew, D., Schelling, X., \& Torres-Ronda, L. (2015). Match activity demands of elite Spanish female basketball players by playing position. International Journal of Performance Analysis in Sport, 15(2), 687-703.

Drinkwater, E. J., Pyne, D. B., \& McKenna, M. J. (2008). Design and interpretation of anthropometric and fitness testing of basketball players. Sports Medicine, 38(7), 565-578.

Edgecomb, S. J., \& Norton, K. I. (2006). Comparison of global positioning and computer-based tracking systems for measuring player movement distance during Australian football. Journal of Science and Medicine in Sport, 9(1), 25-32.

Ferrera, J. C. M., Sánchez, F. J. N., Cabrera, F. I. M., Sánchez, P. R., \& Moreno-Arrones, L. S. (2014). Comparación de las demandas de carrera Futbol 7 vs. Fútbol 11 en jugadores jóvenes de fútbol. Retos. Nuevas Tendencias En Educación Física, Deporte Y Recreación (26), 149-152.

Field, A. (2009). Discovering statistics using SPSS: Sage publications.

Foster, C. D., Twist, C., Lamb, K. L., \& Nicholas, C. W. (2010). Heart rate responses to small-sided games among elite junior rugby league players. The Journal of Strength \& Conditioning Research, 24(4), 906-911.

Gaudino, P., Alberti, G., \& Iaia, F. M. (2014). Estimated metabolic and mechanical demands during different small-sided games in elite soccer players. Human Movement Science, 36, 123-133.

Hernández, D., Casamichana, D., \& Sánchez-Sánchez, J. (2017). La cuantificación de la carga de entrenamiento como estrategia básica de prevención de lesiones. Revista de Preparación Física en el Fútbol, 24(2), 33-39.

Hill-Haas, S., Coutts, A., Rowsell, G., \& Dawson, B. (2009). Generic versus small-sided game training in soccer. International Journal of Sports Medicine, 30(09), 636642.

Hodgson, C., Akenhead, R., \& Thomas, K. (2014). Timemotion analysis of acceleration demands of $4 \mathrm{v} 4$ smallsided soccer games played on different pitch sizes. Human Movement Science, 33, 25-32.

López-Herrero, F., \& Arias-Estero, J. L. (2019). Efecto de la modalidad de juego en baloncesto (5vs. 5 y 3vs. 3) sobre conductas motrices y psicológicas en alumnado de 9-11 años Retos. Nuevas Tendencias En Educación Física, Deporte Y Recreación, 36(36), 354-361

Matthew, D., \& Delextrat, A. (2009). Heart rate, blood lactate concentration, and time-motion analysis of female basketball players during competition. Journal of Sports Sciences, 27(8), 813-821.

McCormick, B. T., Hannon, J. C., Newton, M., Shultz, B., Miller, N., \& Young, W. (2012). Comparison of physical activity in small-sided basketball games versus full-sided games. International Journal of Sports Science \& 
Coaching, 7(4), 689-697.

McInnes, S., Carlson, J., Jones, C., \& McKenna, M. J. (1995). The physiological load imposed on basketball players during competition. Journal of Sports Sciences, 13(5), 387-397.

Metzler, M. (2017). Instructional models in physical education: Taylor \& Francis.

Montgomery, P. G., Pyne, D. B., \& Minahan, C. L. (2010). The physical and physiological demands of basketball training and competition. International Journal of Sports Physiology and Performance, 5(1), 75-86.

Narazaki, K., Berg, K., Stergiou, N., \& Chen, B. (2009). Physiological demands of competitive basketball. Scandinavian Journal of Medicine \& Science in Sports, 19(3), 425-432.

Olthof, S., Frencken, W., \& Lemmink, K. (2017). Match-derived relative pitch area changes the physical and team tactical performance of elite soccer players in small-sided soccer games. Journal of Sports Sciences, 1-7. doi:10.1080/ 02640414.2017.1403412

Ostojic, S. M., Mazic, S., \& Dikic, N. (2006). Profiling in basketball: Physical and physiological characteristics of elite players. Journal of Strength and Conditioning Research, 20(4), 740.

Puente, C., Abián-Vicén, J., Areces, F., López, R., \& Del Coso, J. (2017). Physical and physiological demands of experienced male basketball players during a competitive game. The Journal of Strength \& Conditioning Research, 31(4), 956-962.

Rampinini, E., Impellizzeri, F. M., Castagna, C., Abt, G., Chamari, K., Sassi, A., \& Marcora, S. M. (2007). Factors influencing physiological responses to small-sided soccer games. Journal of Sports Sciences, 25(6), 659-666.

Reina, M., García-Rubio, J., Feu, S., \& Ibáñez, S. J. (2019). Training and Competition Load Monitoring and Analysis of Women's Amateur Basketball by Playing Position: Approach Study. Frontiers in Psychology, 9. http:// doi.org/ 10.3389/fpsyg.2018.02689
Reina, M.; Mancha-Triguero, D.; García-Santos, D.; GarcíaRubio, J., e Ibáñez, Sergio J. (2019). Comparación de tres métodos de cuantificación de la carga de entrenamiento en baloncesto. RICYDE. Revista internacional de ciencias del deporte. 58(15), 368-382. https://doi.org/10.5232/ ricyde2019.05805

Sampaio, J., Abrantes, C., \& Leite, N. (2009). Power, heart rate and perceived exertion responses to $3 \times 3$ and $4 \times 4$ basketball small-sided games. Revista de Psicología del Deporte, 18(3), 463-467.

Sampaio, J., Gonçalves, B., Rentero, L., Abrantes, C., \& Leite, N. (2014). Exploring how basketball players' tactical performances can be affected by activity workload. Science \& Sports, 29(4), e23-e30.

Tee, J. C., Lambert, M. I., \& Coopoo, Y. (2016). GPS comparison of training activities and game demands of professional rugby union. International Journal of Sports Science \& Coaching, 11(2), 200-211.

Thalheimer, W., and Cook, S. (2002). How to calculate effect sizes from published research: a simplified methodology. Work Learn. Res. 1-9. Available online at: http://worklearning.com/effect_sizes.htm

Torres-Ronda, L., Ric, A., Llabres-Torres, I., de Las Heras, B., \& i del Alcazar, X. S. (2016). Position-dependent cardiovascular response and time-motion analysis during training drills and friendly matches in elite male basketball players. The Journal of Strength \& Conditioning Research, 30(1), 60-70.

Travassos, B., Duarte, R., Vilar, L., Davids, K., \& Araujo, D. (2012). Practice task design in team sports: Representativeness enhanced by increasing opportunities for action. Journal of Sports Sciences, 30(13), 1447-1454

White, A. D., \& MacFarlane, N. G. (2015). Analysis of international competition and training in men's field hockey by global positioning system and inertial sensor technology. The Journal of Strength \& Conditioning Research, 29(1), 137-143.
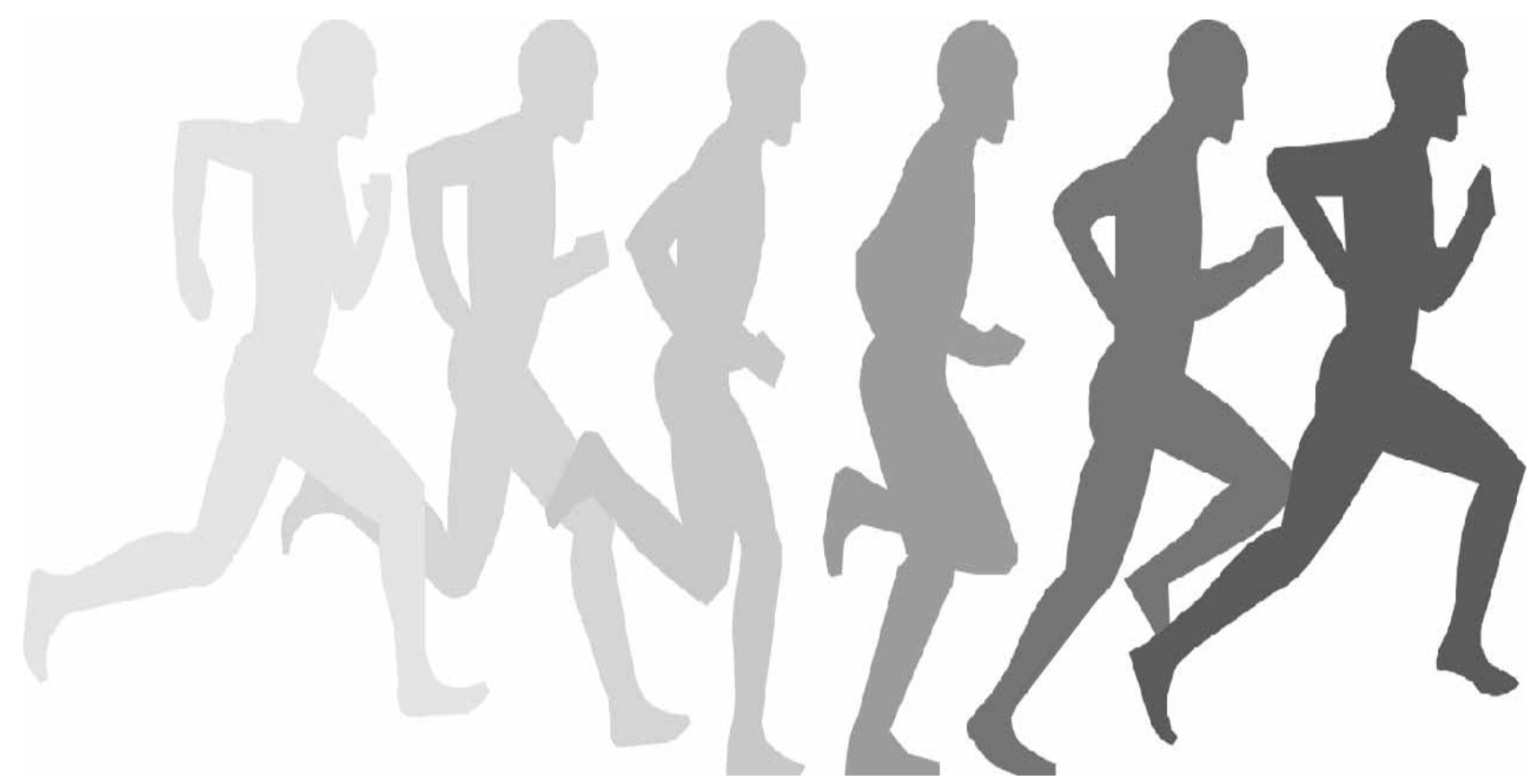